\title{
Concurrent Phase Retrieval for Imaging Strain in Nanocrystals
}

\author{
Marcus C. Newton* \\ Physics 8 Astronomy, University of Southampton, SO17 1BJ, UK
}

(Dated: May 22, 2020)

\begin{abstract}
Coherent diffraction imaging is a form of microscopy that permits high resolution imaging of atomic displacements from equilibrium where the use of conventional optics is not feasible. Approaches to date for the recovery of atomic displacements from equilibrium and subsequently strain information occur after phase reconstruction of the complex real-space images from at least three independent Bragg diffraction amplitude measurements. While this is a more accessible and effective approach to recover strain information, there is potential for erroneous results if the recovered phase information is not carefully treated. Here we present a strategy for imaging strain with coherent $\mathrm{x}$-rays that eliminates the technical challenges that exist in conventional approaches by constructing the strain field concurrently during the phase retrieval process of recovering phase information.
\end{abstract}

\section{INTRODUCTION}

Coherent X-ray diffraction Imaging (CXDI) is a powerful lens-less imaging technique for probing nanometre sized materials with sub-angstrom sensitivity. [1-6] It is an attractive alternative to electron microscopy because of the better penetration of the electromagnetic waves in materials of interest, which are often less damaging to the sample than electrons. There are a number of important variations on the conventional CXDI method. They include curved wavefront Fresnel CXDI [7] and coherent scanning probe ptychography [8, 9].

Bragg Coherent X-ray diffraction Imaging (BCXDI) is the 'classical' CXDI method used to study nanoscale crystals. [6, 10-12] It is performed by exciting a Bragg reflection from the nanocrystal by illuminating a sample with a spatially coherent $\mathrm{x}$-ray source so that the coherence lengths exceed the dimensions of the nanocrystal. This condition can be satisfied by a synchrotron x-ray source. In the Bragg reflection geometry, scattered light from the entire volume of the crystal interferes in the far-field, producing a three-dimensional Fourier-space diffraction pattern. The resulting threedimensional diffraction pattern is then recorded in the far-field Fraunhofer limit. By using small enough detector pixels, a large enough detector distance and fine enough angle steps in the rocking scan, this pattern can be oversampled relative to the Shannon-Nyquist sampling frequency in all three directions. Technically, this operation of measuring a rocking curve with a $2 \mathrm{D}$ detector results in 'binned' (angularly-averaged) data in two directions and 'sampled' data in the third direction that can be inverted using computational algorithms $[13,14]$. The curvature of the Ewald sphere is usually small enough that the speckle pattern can be sampled on a regular grid in all three directions and subsequently transformed in the inversion algorithm using a discrete Fourier transform (Fig. 1).

Along with three-dimensional electron density information, BCXDI also offers a means to determine in

\footnotetext{
*M.C.Newton@soton.ac.uk
}

three-dimensions any structural changes due to strain, most likely to occur for nanocrystals that experience a structural distortion due to defects or external perturbation [12, 15-17]. Ions displaced from the ideal lattice point result in a spatially dependent phase shift of $\phi_{i}(\mathbf{r})=Q_{i j} u_{j}(\mathbf{r})$ in the reflected wave from the distorted region where $u_{j}$ is the local ion displacement vector component set for orthogonal directions $j$, and $i \in \mathbb{N}_{1}$ labels the images obtained at differing $\mathbf{Q}$-vectors. Provided this real-space phase is recovered, a component of the atomic displacement $u_{j}$ is directly obtained in the direction of the $\mathbf{Q}$-vector. Diffraction from multiple Bragg points of the same crystal will in general provide multiple components of the displacement vector from which we can construct the displacement field using the following result [15]:

$$
u_{j}=\xi_{j i} Q_{k i} \phi_{k} ; \quad \xi_{j i}=\left(Q_{k j} Q_{k i}\right)^{-1}
$$

Here $\left(Q_{k j} Q_{k i}\right)^{-1}$ is the inverse matrix of $Q_{k j} Q_{k i}$ (with units of square length). The strain field is directly obtained from the displacement field using the following result:

$$
\epsilon_{i j}=\frac{1}{2}\left(\frac{\partial u_{j}}{\partial x_{i}}+\frac{\partial u_{i}}{\partial x_{j}}\right)
$$

To date, all approaches have obtained the displacement field $u_{i}(\mathbf{r})$ (and subsequently the strain field) after reconstruction of the complex real-space images $\rho_{i}(\mathbf{r})$ (described below) from at least $N=3$ independent Bragg diffraction amplitude measurements $I_{i}(\mathbf{q})$. This equates to the following sequence of tasks:[15] (1) Acquire diffraction data $I_{i}(\mathbf{q})$ for at least three independent Bragg reflections from a single nanocrystal; (2) Reconstruct the diffraction data for each individual Bragg reflection to obtain a separate three-dimensional realspace image $\rho_{i}(\mathbf{r})$ for each Bragg reflection; (3) Combine the phase information $\phi_{i}(\mathbf{r})$ from the complex realspace images $\rho_{i}(\mathbf{r})$ according to equation 1 to obtain the displacement field $u_{i}(\mathbf{r})$, and subsequently strain tensor field $\epsilon_{i j}$.

A number of technical challenges exist with this approach that, if not carefully treated, could lead to erroneous results. The first arises from the inherent properties of project algorithms and the phase reconstruction 
a

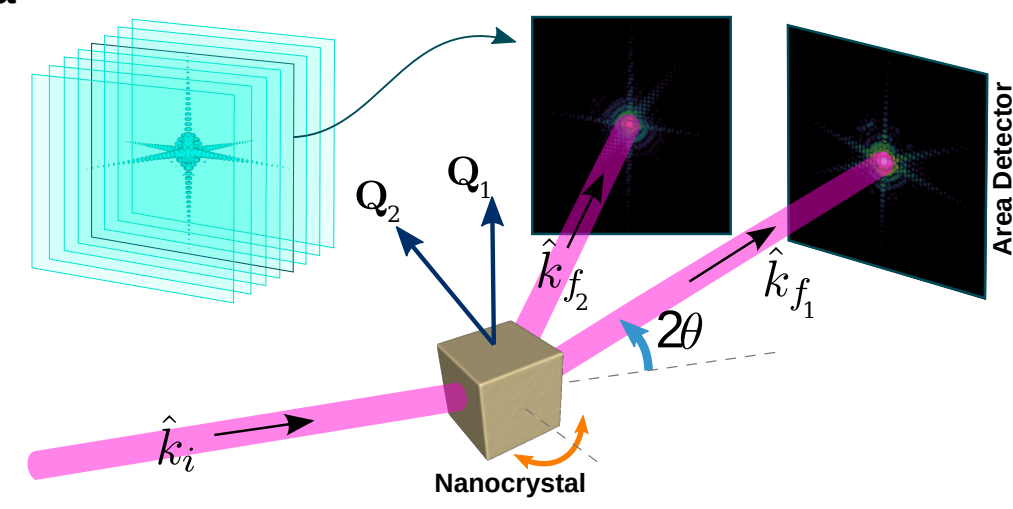

b

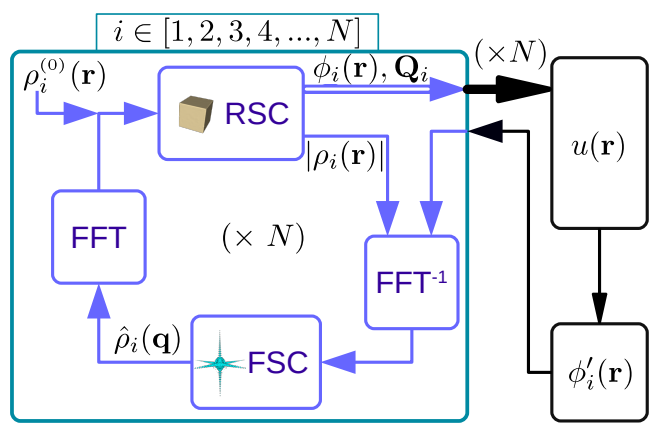

FIG. 1. Diffraction experimental geometry and concurrent phase retrieval method. a, Multiple Bragg reflections with wavevector $\hat{k}_{f_{N}}$ are excited from a single nanocrystal and each speckle pattern acquired (only two are displayed, for clarity). The incident wave vector is denoted as $\hat{k}_{i}$. The rocking curve method is used to acquire two-dimensional slices of the threedimensional speckle pattern by rotating the nanocrystal through the Bragg condition. $\mathbf{b}$, Flow chart of concurrent multiple Bragg reflection method. Enclosed region shows retrieval processes that are common for a single $(N=1)$ Bragg reflection.

problem. As the projection from Fourier-space to realspace is insensitive to translation, each reconstructed image $\rho_{i}(\mathbf{r})$ must first coincide at the same position in real-space so that a given position $\mathbf{r}$ in each image is equivalent. This can be challenging and can lead to artefacts in the strain field if not optimally performed. The second potential issue arises from reconstruction artefacts in amplitude and phase that might appear in a given real-space image $\rho_{i}(\mathbf{r})$ as a consequence of poor convergence or stagnation during phase reconstruction. This can be due to a number of reasons including insufficient sampling in the presence of noise, [18] strong phase structure[19] and dynamic structural changes that can adversely affect speckle visibility. In the following we demonstrate a route to eliminate phase uniqueness issues primarily associated with alignment by reconstructing the displacement field $u_{i}(\mathbf{r})$ concurrently with the phase information contained in images $\rho_{i}(\mathbf{r})$.

\section{EXPERIMENTAL METHODS}

\section{A. Concurrent Phase Retrieval}

When BCXDI measurements are performed phase information is lost and instead we obtain only the reciprocal space scattering intensity distribution $I(\mathbf{q})$ in the form of an interference pattern. This is closely approximated by the square of the modulus of the Fourier transform of the object function $\hat{\rho}_{0}(\mathbf{q})$ such that: $I(\mathbf{q})=\left|\hat{\rho}_{0}(\mathbf{q})\right|^{2}=\left|\hat{\rho}_{0}(\mathbf{q}) e^{i \phi(\mathbf{q})}\right|^{2}$. The third dimension is obtained by rotating the Ewald sphere through the Bragg condition while maintaining a largely fixed incident $\left(\mathbf{k}_{i}\right)$ and reflected $\left(\mathbf{k}_{f}\right)$ wave vector. The $\mathbf{Q}$ vector $\left(\mathbf{Q}=\mathbf{k}_{i}-\mathbf{k}_{f}\right)$ is typically rotated through an angle of $\theta_{\max } \sim 0.5$ angular degrees. The complex real-space image, given by $\rho=|\rho| e^{i \phi(\mathbf{r})}$, is directly pro- portional to the electron density and is recovered using iterative projection algorithms that propagate back and forth between the real-space image plane and the Fourier-space detector plane while applying a constraint at each turn [3, 20-23]. The Fourier-space modulus constraint, $\hat{\boldsymbol{P}}_{\mathrm{M}}$, requires the scattering amplitude to be proportional to the original measurement such that $\hat{\boldsymbol{P}}_{\mathrm{M}}|\hat{\rho}(\mathbf{q})| e^{i \phi(\mathbf{q})}=\left|\hat{\rho}_{0}(\mathbf{q})\right| e^{i \phi(\mathbf{q})}$. The real-space constraint $\boldsymbol{P}_{\mathrm{S}}$ defines a support region where the electron density $\rho(\mathbf{r})$ is unrestricted while all other points are set to zero.

To recover the three-dimensional strain field of a single nanocrystal concurrently, complex real-space images $\rho_{i}(\mathbf{r})$ are reconstructed concurrently from diffraction intensity measurements $I_{i}(\mathbf{q})$. This method proceeds with the following steps (depicted in Figure 1): (1) As before, acquire diffraction data $I_{i}(\mathbf{q})$ for at least $N=3$ independent Bragg reflections from a single nanocrystal; (2) For each iteration of the phase reconstruction process and for each Bragg reflection, propagate the scattering amplitude to the real-space image plane and calculate $u_{i}(\mathbf{r})$ using equation $1 ;(\mathbf{3})$ Use the relationship $\phi_{i}^{\prime}(\mathbf{r})=Q_{i j} u_{j}(\mathbf{r})$ to update the phase information for each Bragg reflection while leaving the amplitude unchanged; (4) Propagate back to the Fourier-space detector plane and apply the amplitude constraint to $\rho_{i}(\mathbf{q})$; Repeat steps (2) to (4) until a sufficiently converged displacement field $\mathbf{u}^{(*)}(\mathbf{r})$ and the final set of images $\rho_{i}^{(*)}(\mathbf{r})$ are obtained. Use the final result for $\mathbf{u}(\mathbf{r})$ to calculate the strain field as shown in equation 2 .

The merit of concurrent phase retrieval for the recovery of strain information is found in its potential to suppress or entirely eliminate artefacts in a reconstructed image that are not consistent with the displacement field obtained using all reconstructed images. This normalising process is not accessible when each complex realspace image is reconstructed independently. 

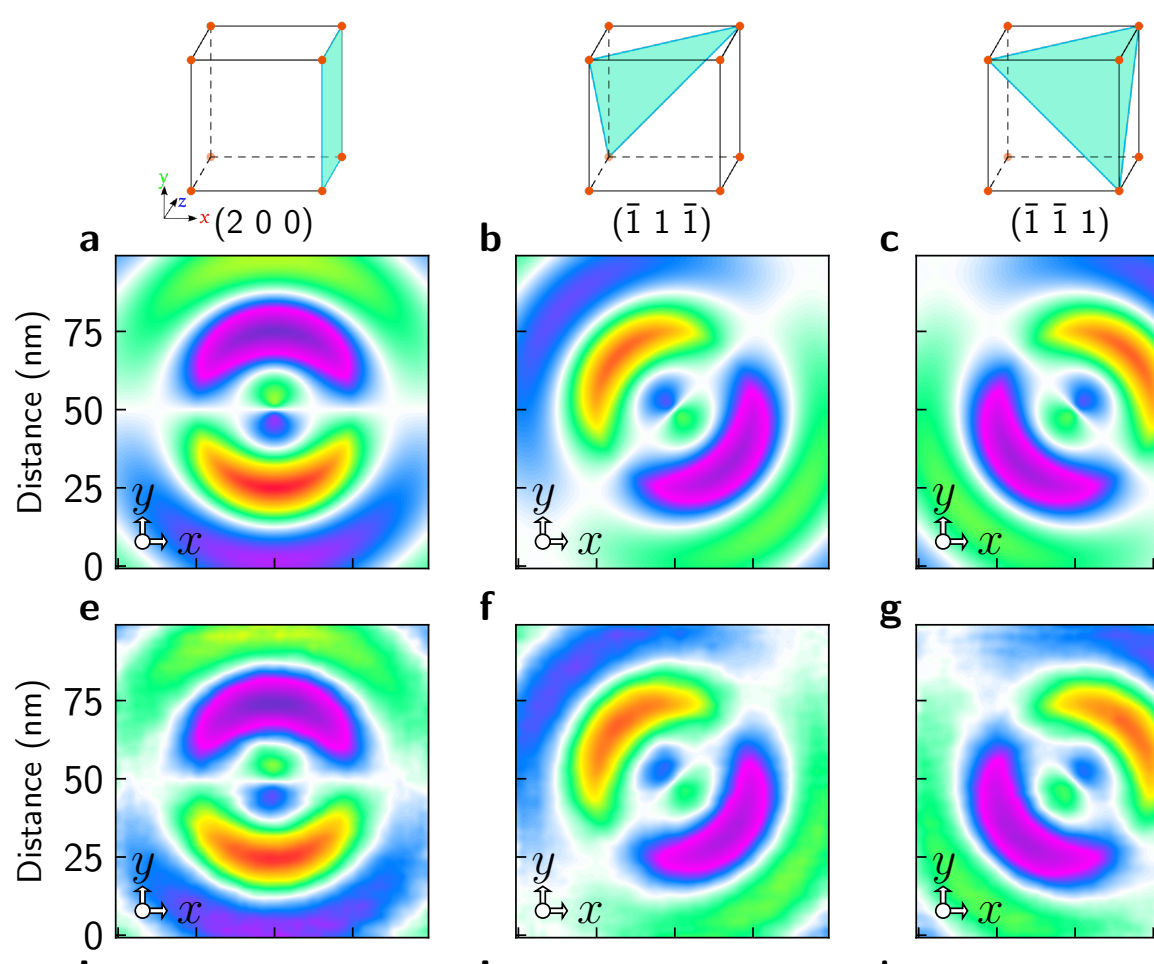

f
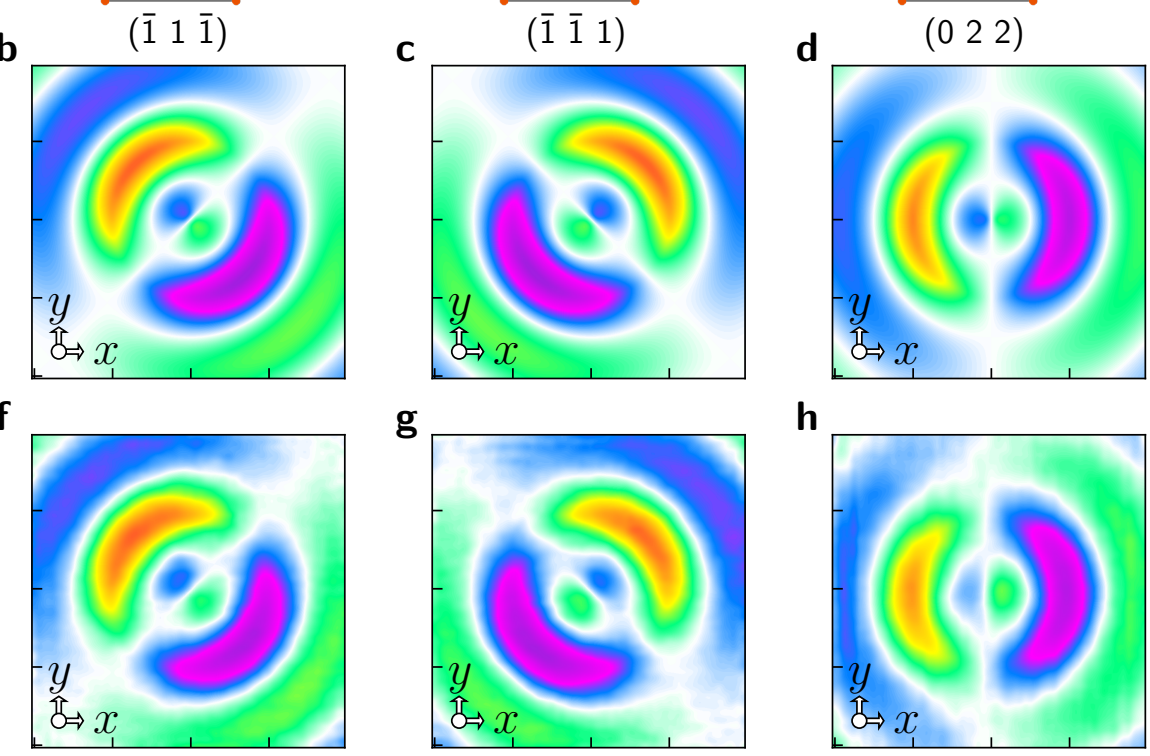

h
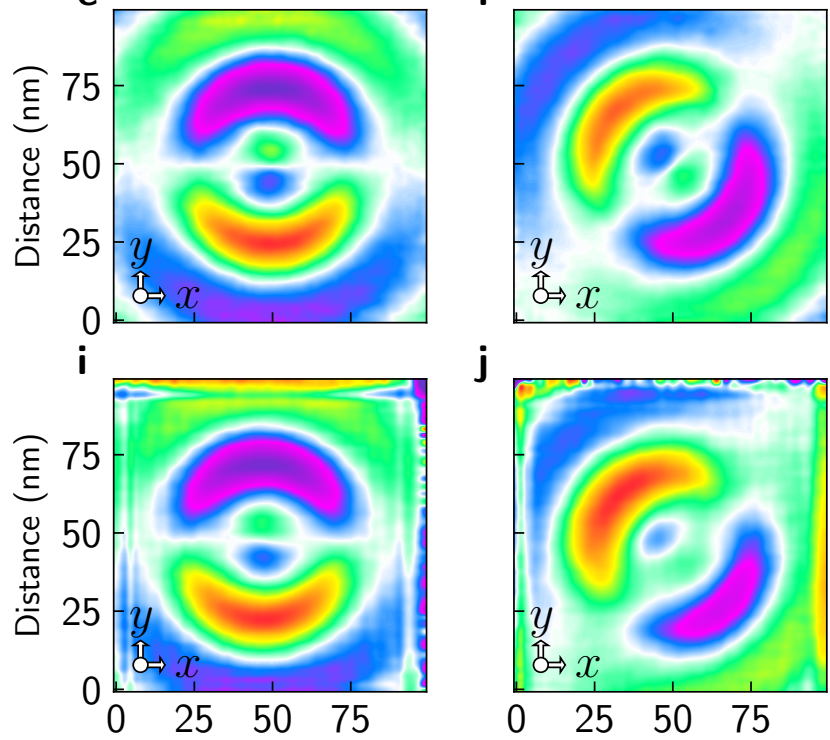

j

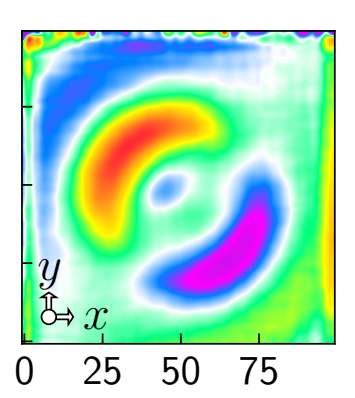

k
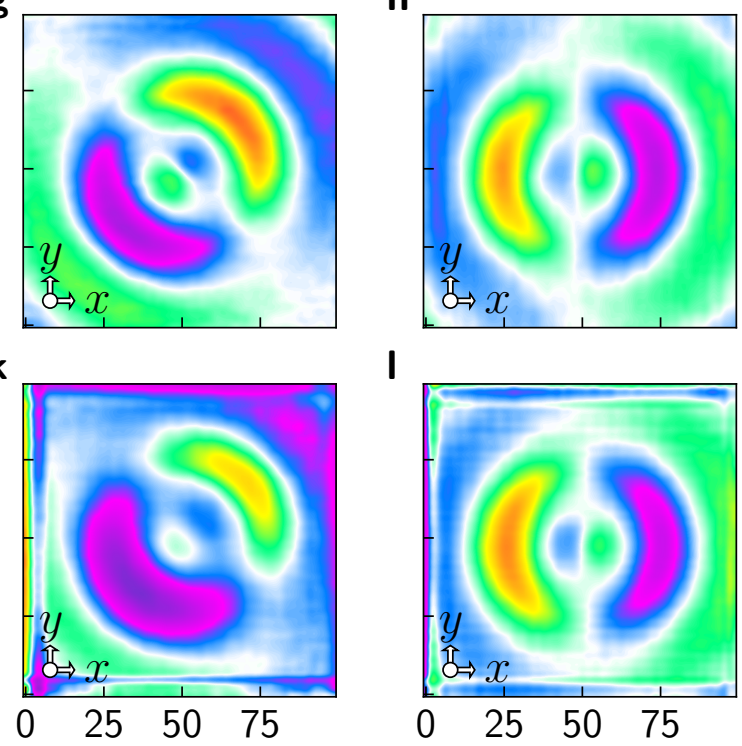

I
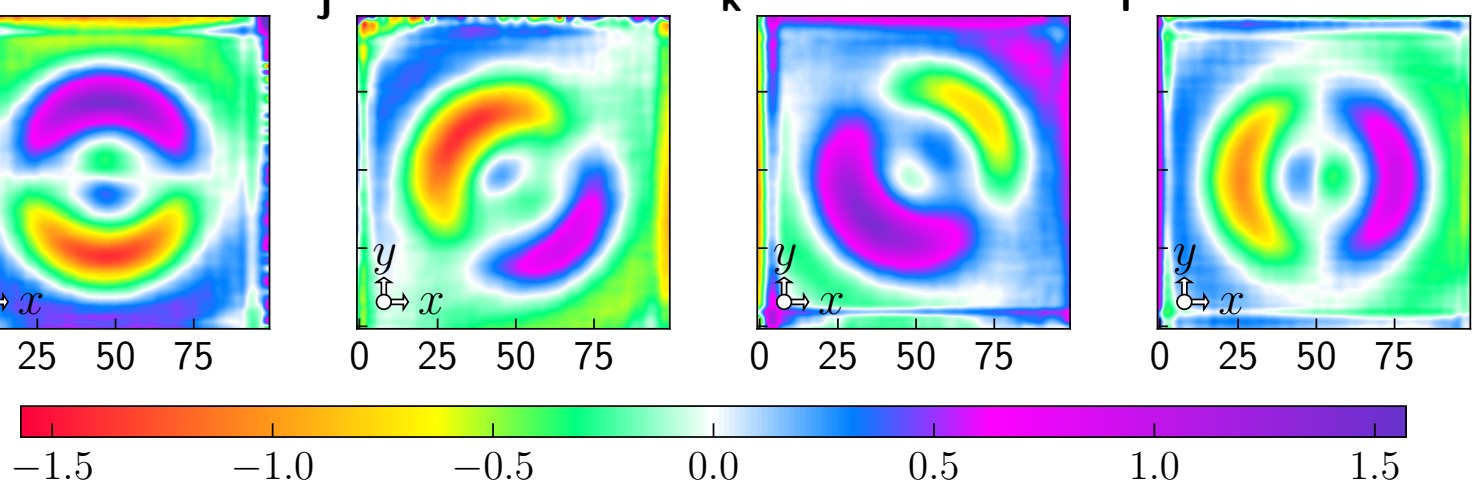

$-0.5$

0.0

0.5

1.0

1.5

Phase (radians)

FIG. 2. Reconstructed phase information for each Bragg reflection. Phase is shown in the $x y$-plane at half distance along the $z$-direction. a-d, Ideal phase profile. e-h, Phase reconstructed concurrently shows good agreement with the ideal case and has few artefacts. i-l, Phase reconstructed independently for each Bragg reflection. The phase is recovered sufficiently well but contains a greater number of phase reconstruction artefacts as this approach does not benefit from the normalising process inherent in the concurrent approach.

\section{RESULTS AND DISCUSSIONS}

\section{A. Experimental procedure}

To determine the efficacy of the concurrent phase retrieval technique, we performed a simulated BCXDI experiment on a single nanocube to record the scattering intensity from multiple Bragg reflections. We subsequently reconstructed the strain tensor field information using both the concurrent and conventional independent phase reconstruction methods under equivalent conditions for comparison. Numerical experiments were performed on a well faceted nanocube with $x=100 \mathrm{~nm}$ side lengths and well defined atomic displacement field information (see Appendix A). An isolated nanocube was initially oriented to the (200), ( $11 \overline{1}),(\overline{11} 1)$ and (022) Bragg reflections. In each case, the nanocube was illuminated with monochromatic x-rays at a wavelength of $8 \mathrm{keV}$ and a three-dimensional far-field intensity speckle pattern was recorded using the rockingcurve method with an oversampling ratio of 2 relative to the Shannon-Nyquist sampling frequency. In the kinematic approximation, the far-field diffraction intensity of the nanocubes electron density is well approximated by the square modulus of the Fourier after applying 

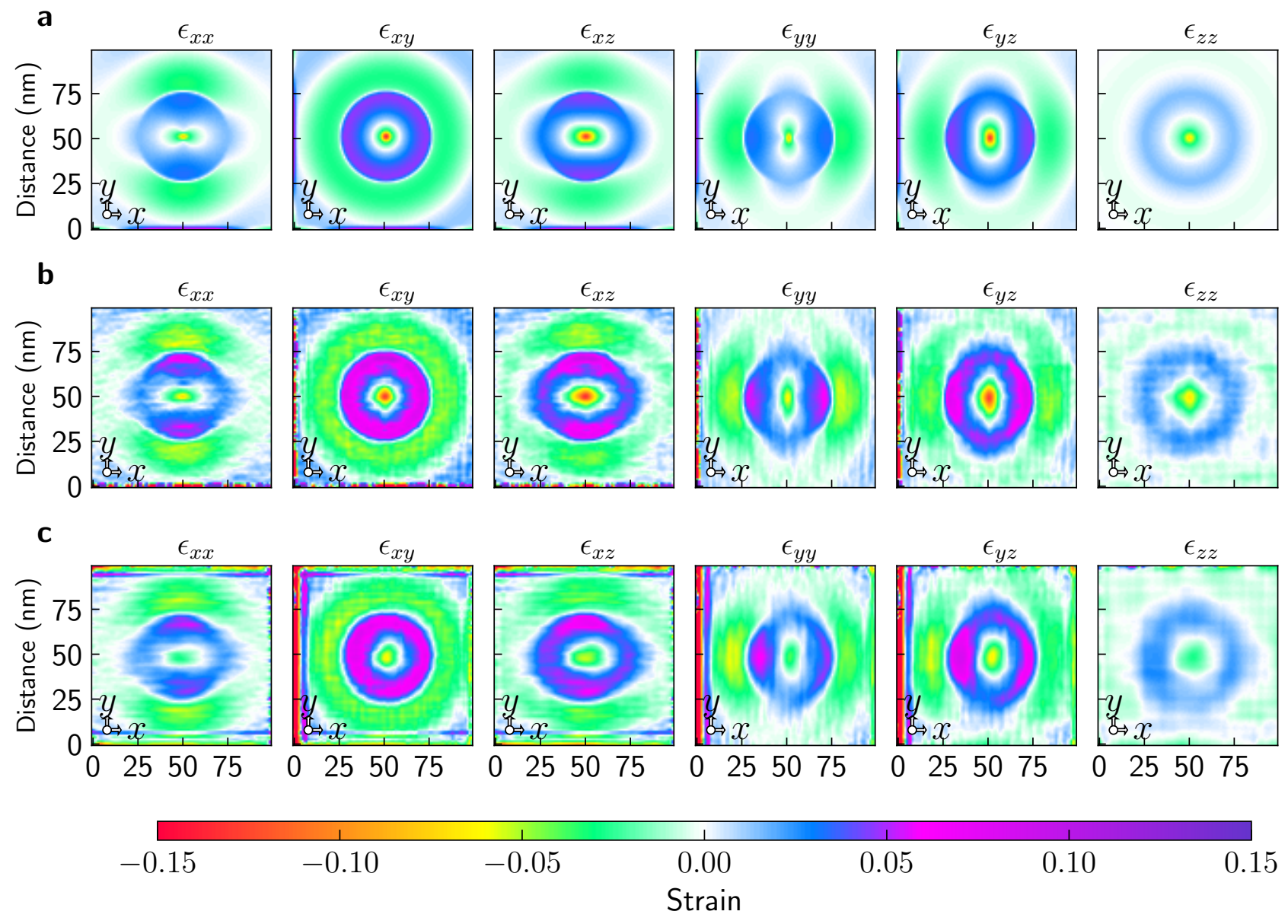

FIG. 3. Two-dimensional slices through the Strain tensor information shown for the ideal, concurrent and independent phase reconstruction methods. Strain is shown in the $x y$-plane at half distance along the $z$-direction. a, Ideal strain tensor information. b, Strain tensor information reconstructed concurrently shows good agreement with the ideal case. c, Strain tensor information reconstructed independently for each Bragg reflection shows reasonable agreement with the ideal case. Artefacts such as ripples in the phase which can occur as a results of sub-optimal alignment are visible in the independently reconstructed phase information.

corrections specific to the experimental geometry. [2428] Utilising the cubic symmetry of the nanocube of side length $x$, we can define the fringe periodicity as $w_{f}=2 \pi / x$ in units of $\mathrm{m}^{-1}$. The rocking-curve angular increment is given by $\delta \theta=w_{f} /(2 \beta|\mathbf{Q}|)$ where $\beta$ is the oversampling ratio.

The recorded speckle patterns were subsequently transformed onto a regular grid (see Appendix B) and interpolated as needed to ensure that correspondence between data points for each speckle pattern. [29, 30] Poisson noise was added to each speckle pattern of amount $\sigma_{A}=5 \%, 10 \%, 20 \%$ and $50 \%$ where:

$$
\sigma_{A}=\frac{\sum_{\mathbf{q}}\left\|A(\mathbf{q})|-| A^{\prime}(\mathbf{q})\right\|}{\sum_{\mathbf{q}}|A(\mathbf{q})|}
$$

and $A^{\prime}(q)$ is the scattering amplitude with Poisson noise applied (See Appendix B). The recorded speckle patterns were then used to recover the real-space com- plex electron density images along with the strain information using both the concurrent and conventional independent methods of phase retrieval. In all cases, reconstruction was carried out using 10,000 iterations of Fienup's hybrid input-output (HIO) algorithm with a relaxation parameter of $\beta=0.9$. Reconstruction proceeded with random phase information and was repeated 10 times to confirm reproducibility.

\section{B. Recovery of Strain Information}

Figure 2 shows a cut plane of the reconstructed phase information of the complex real-space images $\rho_{i}(\mathbf{r})$ obtained using the concurrent $(\mathbf{e}-\mathbf{h})$ and conventional independent (i-l) phase reconstruction methods.[31] The recovered phase is contrasted with the ideal case $(\mathbf{a}-\mathbf{d})$ for direct comparison. Phase information recovered using the concurrent methods shows good agreement with 
TABLE I. Standard deviation $\sigma$ of the phase difference $\Delta \phi(\mathbf{r})$ between the ideal and recovered phase in the presence of Poisson noise $\sigma_{A}$.

\begin{tabular}{c|cccc|cccc}
\hline Noise & \multicolumn{4}{|c|}{ Concurrent $\sigma$} & \multicolumn{4}{c}{ Independent $\sigma$} \\
$\sigma_{A}$ & $(200)$ & $(\overline{1} 1 \overline{1})$ & $(\overline{11} 1)$ & $(022)$ & $(200)$ & $(\overline{1} 1 \overline{1})$ & $(\overline{11} 1)$ & $(022)$ \\
\hline \hline $0 \%$ & 1.11 & 1.15 & 1.16 & 1.68 & 1.61 & 1.60 & 1.61 & 1.62 \\
$5 \%$ & 1.14 & 1.28 & 1.28 & 1.61 & 1.61 & 1.61 & 1.61 & 1.59 \\
$10 \%$ & 1.16 & 1.28 & 1.28 & 1.60 & 1.63 & 1.59 & 1.60 & 1.62 \\
$20 \%$ & 1.16 & 1.29 & 1.28 & 1.63 & 1.61 & 1.61 & 1.60 & 1.62 \\
$50 \%$ & 1.16 & 1.30 & 1.30 & 1.63 & 1.61 & 1.59 & 1.56 & 1.60 \\
\hline \hline
\end{tabular}

the ideal case with only minor deviations visible. All 10 attempts at recovering the phase information showed good agreement with the ideal case and displayed no appreciable differences. Phase information recovered independently shows reasonable agreement with the ideal case but with an increase in artefacts likely due to stagnation of the HIO algorithm as is known to occur for unidirectional saddle-point optimisation methods.[21, 32]

Figure 3 shows a cut plane of the reconstructed strain information obtained using the concurrent $(\mathbf{e}-\mathbf{h})$ and conventional independent (i-l) phase reconstruction methods. Strain information recovered using the concurrent methods shows good agreement with the ideal case with each tensor component showing comparable characteristic features. Phase information recovered independently shows some agreement but deviations from the ideal case are clearly seen, particularly in the $\epsilon_{x y}$, $\epsilon_{x z}$ and $\epsilon_{y z}$ components. This is to be expected as tensor cross terms are more sensitive to misalignment of the phase components from each Bragg reflection.

Table I hows the standard deviation of the phase difference $\Delta \phi$ between the ideal and recovered phase in the presence of Poisson noise $\sigma_{A}$ (Equation 3). In each case, the concurrently recovered phase generally has a lower value of standard deviation than the independently recovered phase. This implies greater agreement with the ideal phase due the suppression of artefacts which persist in the independently reconstructed phase information.[33]

Figure 4 shows the mean error residual (of each independent intensity measurements $I_{i}(\mathbf{q})$ ) obtained for the concurrent and conventional independent phase reconstruction methods plotted against the respective iteration. The error metric is defined as[22, 23]:

$$
\begin{aligned}
\varepsilon(\rho) & =\varepsilon_{\mathrm{M}}^{2}(\rho)-\varepsilon_{\mathrm{S}}^{2}(\rho) \\
\varepsilon_{\mathrm{M}} & =\left\|\boldsymbol{P}_{\mathrm{M}} \rho-\rho\right\| \\
\varepsilon_{\mathrm{S}} & =\left\|\boldsymbol{P}_{\mathrm{S}} \rho-\rho\right\|
\end{aligned}
$$

where $\boldsymbol{P}_{\mathrm{M}}=\mathcal{F} \hat{\boldsymbol{P}}_{\mathrm{M}} \mathcal{F}^{-1}$ and $\mathcal{F}$ is the Fourier transform. While both the concurrent and conventional independent phase reconstruction methods show good convergence, the concurrent methods shows consistently

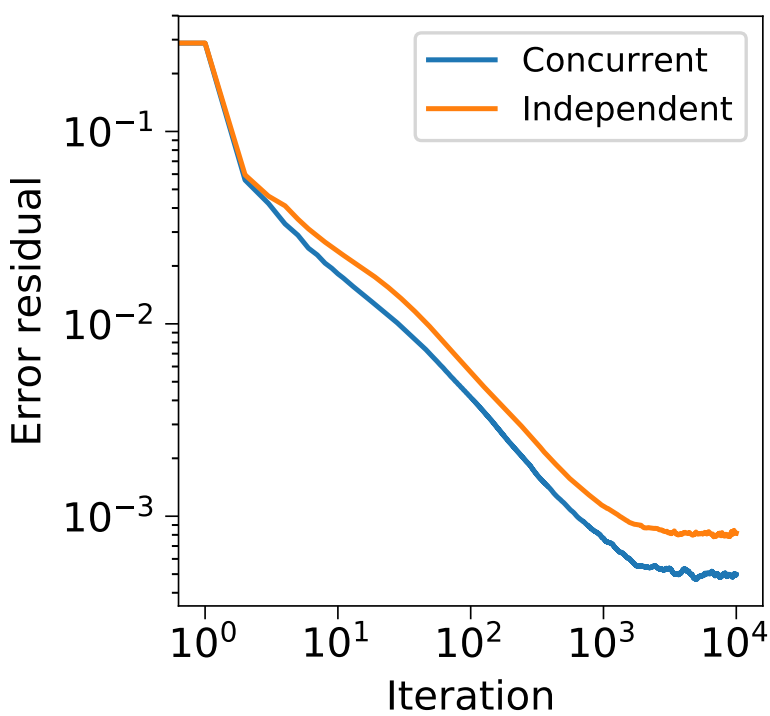

FIG. 4. Error residual plotted for the concurrent and conventional independent phase reconstruction methods. The error plotted is the mean error for each Bragg reflection at a given iteration. The concurrent approach clearly shows stronger convergence down to $5 \times 10^{-4}$ while the independent phase reconstruction method was found to stagnate at just above $10^{-3}$.

stronger convergence at a given iteration and converges below $10^{-3}$ down to $5 \times 10^{-4}$ while the independent phase reconstruction method was found to stagnate at just above $10^{-3}$. In addition, this was found to occur for all attempts at reconstruction staring with random phase suggesting a route to deterministic phase retrieval.

\section{CONCLUSION}

Concurrent phase retrieval provides a route to the reconstruction of complex real-space images $\rho_{i}(\mathbf{r})$ and their phase information $\phi_{i}(\mathbf{r})$ that are inherently consistent. Our findings have shown that reconstructing strain tensor information concurrently avoids many of the pitfall of independent phase reconstruction such as phase artefacts. In addition twinned objects, i.e. those with conjugated image $\rho^{*}(-\mathbf{r})$ but equal Fourier amplitude to the image $\rho(\mathbf{r})$, are also avoided inherently as their phase information cannot consistently combine with un-conjugated images during phase retrieval. This suggests that recovering phase information concurrently could provide a route to deterministic phase retrieval and strain field reconstruction.

The approach demonstrated herein will also greatly facilitate the reconstruction of strain field information from materials with phase information that is challenging to reconstruct. Examples include highly strained materials or those undergoing a structural transformation from one crystal structure to another. There is also 
potential to further refine the above method by utilising additional algorithms including error reduction, higher dimensional saddle point optimisation and accounting for partial coherence in the Fourier-space modulus constraint $[22,34,35]$. In addition, recent advances in machine learned iterative deep neural networks that encode high-level information about the class of images could also facilitate in mitigating artefacts during reconstruction [18].

\section{Appendix A: BCXDI experimental setup}

Nanocubes with the face-centred cubic structure of silver metal and side lengths of $x=100 \mathrm{~nm}$ were constructed with displacement field information given by:

$$
\begin{aligned}
\mathbf{u}(\mathbf{r}) & =\frac{\cos \left(\pi s / d_{1}\right)}{2\left(|s| / d_{2}+1\right)^{2}} \hat{\mathbf{r}} \\
s & =\left(|\mathbf{r}|-d_{1}\right)
\end{aligned}
$$

where $d_{1}=x / 4$ is a displacement of a quarter distance of the nanocube length and $d_{2}=x / 8$ is a displacement of an eighth distance of the nanocube length. With coherent x-ray illumination in the far-field Fraunhofer limit, the scattering amplitude is given by:

$$
A(\mathbf{q})=\int \mathrm{d} \mathbf{r} \rho(\mathbf{r}) \exp (\mathrm{iq} \cdot \mathbf{r}) \exp (\mathrm{iq} \cdot \mathbf{u}(\mathbf{r}))
$$

from which we measure the intensity $I(\mathbf{q})=A^{*}(\mathbf{q}) A(\mathbf{q})$. $\delta \alpha=w_{f} /(2 \beta|\mathbf{k}|)$ is defined as the angle subtending a single detector pixel $d_{x, y}$ for an oversampling ratio of $\beta$. From this we can directly obtain the distance from the sample to the detector as $R=d_{x, y} / \delta \alpha$. This condition ensures that each sampling interval is acquired by a single pixel on the detector. The maximum intensity per pulse per $\mathrm{m}^{2}$ at the center pixel is given by $I_{s c}=r_{0}^{2} \Phi_{0} F^{2} N^{2} / R^{2}$, where $r_{0}$ is the Thomson scattering length and $F$ is the unit cell structure factor. The envelope function of the scattering intensity is given by $I(q)=I_{s c} /\left(\left(\pi q / w_{f}\right)^{2}+1\right)$ and takes its minimum value when $I\left(q_{\max }\right)=n_{0} / d_{x, y}^{2}$, where $n_{0}$ is the minimum photon count a detector pixel can detect. The total rocking width is then given by

$$
\theta_{\max }=\frac{2 w_{f}}{|\mathbf{Q}| \pi}\left(\frac{I_{s c} d_{x, y}^{2}}{n_{0}}-1\right)^{1 / 2}
$$

and the resolution is $\Delta x=2 \pi /|\mathbf{Q}| \theta_{\max }$.

Using $8 \mathrm{keV}$ x-rays with a photon flux density of $9.3 \times 10^{21}$ photons $/ \mathrm{m}^{2}$, the resulting speckle pattern is sampled onto a photon counting detector with $50 \times 50$ $\mathrm{\mu m}^{2}$ pixels and an oversampling ratio of 2 is maintained at a sample-to-detector propagation distance of approximately $0.14 \mathrm{~m}$. Real-space images were reconstructed using intensity information above 5 photon counts per second and recovered with a resolution of approximately 4.5 nanometres.

\section{Appendix B: Diffraction Pattern Coordinate Transformation}

Diffraction amplitude measurements are in general recorded in the coordinate system of the experimental setup which is typically not a rectilinear coordinate system. As a result, the amplitude and phase information reconstructed from diffraction amplitude measurements will also not reside in a rectilinear coordinate system. It is however possible to transform the experimental coordinate system into a rectilinear coordinate system. [30] We demonstrate this using the following procedure for a $\theta$ rocking curve measurement. Analogous procedures apply for a $\phi$ rocking curve measurement. If $\hat{\mathbf{i}}, \hat{\mathbf{j}}$ and $\hat{\mathbf{k}}$ are unit vectors in a rectilinear coordinate system, unit vectors for the incident $\mathbf{k}_{i}$ and reflected $\mathbf{k}_{f}$ wavevectors are generally given by:

$$
\begin{aligned}
\hat{\mathbf{k}}_{i}= & \{\cos (\phi) \sin (\theta)\} \hat{\mathbf{i}} \\
& +\sin (\phi) \hat{\mathbf{j}} \\
& +\{\cos (\phi) \cos (\theta)\} \hat{\mathbf{k}} \\
\hat{\mathbf{k}}_{f}= & \left\{\cos \left(\phi+\phi_{0}+\phi_{0}^{\prime}\right) \sin \left(\theta+\theta_{0}+\theta_{0}^{\prime}\right)\right\} \hat{\mathbf{i}} \\
& +\sin \left(\phi+\phi_{0}+\phi_{0}^{\prime}\right) \hat{\mathbf{j}} \\
& +\left\{\cos \left(\phi+\phi_{0}+\phi_{0}^{\prime}\right) \cos \left(\theta+\theta_{0}+\theta_{0}^{\prime}\right)\right\} \hat{\mathbf{k}}
\end{aligned}
$$

where $\theta$ and $\phi$ are angular rotations about the $\hat{\mathbf{j}}$ and $\hat{\mathbf{i}}$ axes respectively. This system of rotations is chosen so that the $\hat{\mathbf{k}}_{i}$ unit vector is aligned along the $\hat{\mathbf{k}}$ vector direction in the absence of rotation. Angles $\theta_{0}$ and $\phi_{0}$ are additional angular rotations about the $\hat{\mathbf{j}}$ and $\hat{\mathbf{i}}$ axes respectively needed for the $\mathbf{k}_{f}$ vector to adopt the Bragg condition for diffraction. Angle $\theta_{0}^{\prime}$ and $\phi_{0}^{\prime}$ are small increments of $\theta_{0}$ and $\phi_{0}$ respectively which allow each point on the diffraction pattern to be addressed by a general rotation of the $\mathbf{k}_{f}$ vector about the origin. The unit $\mathbf{Q}$-vector is then given by:

$$
\hat{\mathbf{Q}}=\frac{\mathbf{k}_{f}-\mathbf{k}_{i}}{\left|\mathbf{k}_{f}-\mathbf{k}_{i}\right|}
$$

In order to define a rectilinear coordinate system, we determine, with zero order approximation, the change in $\hat{\mathbf{Q}}$ with, $\theta_{0}^{\prime}, \phi_{0}^{\prime}$ and $\theta$. This is because the rocking-curve rotates about $\hat{\mathbf{Q}}$.

$$
\begin{aligned}
\frac{\partial \hat{\mathbf{Q}}}{\partial \theta_{0}^{\prime}}= & \left\{\cos \left(\phi_{0}\right) \cos \left(\theta_{0}\right)\right\} \hat{\mathbf{i}} \\
& -\left\{\cos \left(\phi_{0}\right) \sin \left(\theta_{0}\right)\right\} \hat{\mathbf{k}} \\
\frac{\partial \hat{\mathbf{Q}}}{\partial \phi_{0}^{\prime}}= & -\left\{\sin \left(\phi_{0}\right) \sin \left(\theta_{0}\right)\right\} \hat{\mathbf{i}} \\
& +\cos \left(\phi_{0}\right) \hat{\mathbf{j}} \\
& -\left\{\sin \left(\phi_{0}\right) \cos \left(\theta_{0}\right)\right\} \hat{\mathbf{k}}
\end{aligned}
$$




$$
\begin{aligned}
\frac{\partial \hat{\mathbf{Q}}}{\partial \theta}= & \left\{\cos \left(\phi_{0}\right) \cos \left(\theta_{0}\right)-1\right\} \hat{\mathbf{i}} \\
& -\left\{\cos \left(\phi_{0}\right) \sin \left(\theta_{0}\right)\right\} \hat{\mathbf{k}}
\end{aligned}
$$

It is then possible to define reciprocal lattice vectors $\mathbf{a}^{*}, \mathbf{b}^{*}$ and $\mathbf{c}^{*}$ in a rectilinear coordinate system as fol- lows:

$$
\begin{aligned}
& \mathbf{a}^{*}=\left|\mathbf{k}_{f}\right| \Delta \phi_{0} \frac{\partial \hat{\mathbf{Q}}}{\partial \phi_{0}^{\prime}} \\
& \mathbf{b}^{*}=\left|\mathbf{k}_{f}\right| \Delta \theta_{0} \frac{\partial \hat{\mathbf{Q}}}{\partial \theta_{0}^{\prime}} \\
& \mathbf{c}^{*}=\left|\mathbf{k}_{f}\right| \Delta \theta \frac{\partial \hat{\mathbf{Q}}}{\partial \theta}
\end{aligned}
$$

where $\Delta \theta$ are angular increments in the rocking-curve measurement and $\Delta \theta_{0}, \Delta \phi_{0}$ are angular displacements of the $\hat{\mathbf{k}}_{f}$ vector. The coordinates generated will in general differ for each speckle pattern that is obtained from a unique Bragg reflection. In order to generate a coordinate points common to all speckle patterns, it is in general necessary to interpolate onto a regular grid.[29]
[1] J. Miao, T. Ishikawa, I. K. Robinson, and M. M. Murnane, Beyond crystallography: Diffractive imaging using coherent x-ray light sources, Science 348, 530 (2015).

[2] J. Miao, P. Charalambous, J. Kirz, and D. Sayre, Extending the methodology of x-ray crystallography to allow imaging of micrometre-sized non-crystalline specimens, Nature 400, 342 (1999).

[3] D. Sayre, Some implications of a theorem due to shannon, Acta Crystallographica 5, 843 (1952).

[4] H. N. Chapman, A. Barty, S. Marchesini, A. Noy, S. P. Hau-Riege, C. Cui, M. R. Howells, R. Rosen, H. He, J. C. H. Spence, U. Weierstall, T. Beetz, C. Jacobsen, and D. Shapiro, High-resolution ab initio threedimensional x-ray diffraction microscopy, J. Opt. Soc. Am. A 23, 1179 (2006).

[5] S. Marchesini, H. N. Chapman, S. P. Hau-Riege, R. A. London, A. Szoke, H. He, M. R. Howells, H. Padmore, R. Rosen, J. C. H. Spence, and U. Weierstall, Coherent $\mathrm{x}$-ray diffractive imaging: applications and limitations, Opt. Express 11, 2344 (2003).

[6] M. A. Pfeifer, G. J. Williams, I. A. Vartanyants, R. Harder, and I. K. Robinson, Three-dimensional mapping of a deformation field inside a nanocrystal, Nature 442, 63 (2006).

[7] K. A. Nugent, Coherent methods in the x-ray sciences, Advances in Physics 59, 1 (2010).

[8] J. M. Rodenburg and H. M. L. Faulkner, A phase retrieval algorithm for shifting illumination, Applied Physics Letters 85, 4795 (2004).

[9] J. M. Rodenburg, A. C. Hurst, A. G. Cullis, B. R. Dobson, F. Pfeiffer, O. Bunk, C. David, K. Jefimovs, and I. Johnson, Hard-x-ray lensless imaging of extended objects, Phys. Rev. Lett. 98, 034801 (2007).

[10] J. Miao, J. Kirz, and D. Sayre, The oversampling phasing method, Acta Crystallographica Section D 56, 1312 (2000).

[11] I. Robinson and J. Miao, Three-dimensional coherent x-ray diffraction microscopy, MRS Bulletin 29, 177 (2004).

[12] I. Robinson and R. Harder, Coherent x-ray diffraction imaging of strain at the nanoscale, Nature Materials $\mathbf{8}$, 291 (2009).
[13] C. Song, D. Ramunno-Johnson, Y. Nishino, Y. Kohmura, T. Ishikawa, C.-C. Chen, T.-K. Lee, and J. Miao, Phase retrieval from exactly oversampled diffraction intensity through deconvolution, Phys. Rev. B 75, 012102 (2007).

[14] R. H. T. Bates, Fourier phase problems are uniquely solvable in more than one dimension i: Underlying theory, Optik 61, 247 (1982).

[15] M. C. Newton, S. J. Leake, R. Harder, and I. K. Robinson, Three-dimensional imaging of strain in a single zno nanorod, Nature Materials 9, 120 (2010).

[16] S. Labat, M.-I. Richard, M. Dupraz, M. Gailhanou, G. Beutier, M. Verdier, F. Mastropietro, T. W. Cornelius, T. U. Schülli, J. Eymery, and O. Thomas, Inversion domain boundaries in gan wires revealed by coherent bragg imaging, ACS Nano 9, 9210 (2015), https://doi.org/10.1021/acsnano.5b03857.

[17] F. Hofmann, N. W. Phillips, R. J. Harder, W. Liu, J. N. Clark, I. K. Robinson, and B. Abbey, Micro-beam Laue alignment of multi-reflection Bragg coherent diffraction imaging measurements, Journal of Synchrotron Radiation 24, 1048 (2017).

[18] J. Adler and O. Öktem, Solving ill-posed inverse problems using iterative deep neural networks, Inverse Problems 33, 124007 (2017).

[19] X. Huang, R. Harder, G. Xiong, X. Shi, and I. Robinson, Propagation uniqueness in three-dimensional coherent diffractive imaging, Phys. Rev. B 83, 224109 (2011).

[20] R. W. Gerchberg and W. O. Saxton, A practical algorithm for the determination of phase from image and diffraction plane apertures, Optik 35, 237 (1972).

[21] J. Fienup, Phase retrieval algorithms - a comparison, Applied Optics 21, 2758 (1982).

[22] S. Marchesini, Phase retrieval and saddle-point optimization, J. Opt. Soc. Am. A 24, 3289 (2007).

[23] H. Stark, New York: Academic Press, 1987, edited by Stark, Henry (Academic Press, 1987).

[24] A. L. Patterson, The diffraction of x-rays by small crystalline particles, Phys. Rev. 56, 972 (1939).

[25] I. Robinson and I. Vartanyants, Use of coherent x-ray diffraction to map strain fields in nanocrystals, Applied Surface Science 182, 186 (2001), proceedings of the International Workshop on Nanomaterials. 
[26] H. Öztürk, X. Huang, H. Yan, I. K. Robinson, I. C. Noyan, and Y. S. Chu, Performance evaluation of bragg coherent diffraction imaging, New Journal of Physics 19, 103001 (2017).

[27] J. Als-Nielsen and D. McMorrow, Elements of Modern X-Ray Physics (Wiley, 2011).

[28] A. G. Shabalin, O. M. Yefanov, V. L. Nosik, V. A. Bushuev, and I. A. Vartanyants, Dynamical effects in bragg coherent x-ray diffraction imaging of finite crystals, Phys. Rev. B 96, 064111 (2017).

[29] D. Shepard, A two-dimensional interpolation function for irregularly-spaced data, in Proceedings of the 1968 23rd ACM National Conference, ACM '68 (Association for Computing Machinery, New York, NY, USA, 1968) p. $517-524$.

[30] M. A. Pfeifer, Structural Studies of Lead Nanocrystals
Using Coherent X-ray Diffraction, Ph.D. thesis, University of Illinois (2005).

[31] See supplementary material at [url will be inserted by publisher] for additional cut planes of the reconstructed phase information.

[32] J. R. Fienup and C. C. Wackerman, Phase-retrieval stagnation problems and solutions, J. Opt. Soc. Am. A 3, 1897 (1986).

[33] See supplementary material at [url will be inserted by publisher] for corresponding phase images.

[34] M. C. Newton, X. Shi, U. Wagner, and C. Rau, Coherent diffraction imaging of a progressively deformed nanocrystal, Phys. Rev. Materials 3, 043803 (2019).

[35] J. N. Clark, X. Huang, R. Harder, and I. K. Robinson, High-resolution three-dimensional partially coherent diffraction imaging, Nature Communications 3, 993 (2012), article. 